\title{
Fast and Efficient Rmap Assembly Using the Bi-Labelled de Bruijn Graph
}

Kingshuk Mukherjee

Department of Computer and Information Science and Engineering, Herbert Wertheim College of Engineering, University of Florida, Gainesville, USA

kingufl@ufl.edu

\section{Massimiliano Rossi}

Department of Computer and Information Science and Engineering, Herbert Wertheim College of Engineering, University of Florida, Gainesville, USA

rossi.m@ufl.edu

\section{Leena Salmela}

Department of Computer Science, Helsinki Institute for Information Technology, University of Helsinki, Finland

leena.salmela@cs.helsinki.fi

\section{Christina Boucher}

Department of Computer and Information Science and Engineering, Herbert Wertheim College of Engineering, University of Florida, Gainesville, USA

christinaboucher@ufl.edu

\begin{abstract}
Genome wide optical maps are high resolution restriction maps that give a unique numeric representation to a genome. They are produced by assembling hundreds of thousands of single molecule optical maps, which are called Rmaps. Unfortunately, there exists very few choices for assembling Rmap data. There exists only one publicly-available non-proprietary method for assembly and one proprietary method that is available via an executable. Furthermore, the publicly-available method, by Valouev et al. (2006), follows the overlap-layout-consensus (OLC) paradigm, and therefore, is unable to scale for relatively large genomes. The algorithm behind the proprietary method, Bionano Genomics' Solve, is largely unknown. In this paper, we extend the definition of bi-labels in the paired de Bruijn graph to the context of optical mapping data, and present the first de Bruijn graph based method for Rmap assembly. We implement our approach, which we refer to as RMAPPER, and compare its performance against the assembler of Valouev et al. (2006) and Solve by Bionano Genomics on data from three genomes - E. coli, human, and climbing perch fish (Anabas Testudineus). Our method was the only one able to successfully run on all three genomes. The method of Valouev et al.(2006) only successfully ran on $E$. coli and Bionano Solve successfully ran on E. coli and human but not on the fish genome. Moreover, on the human genome RMAPPER was at least 130 times faster than Bionano Solve, used five times less memory and produced the highest genome fraction with zero mis-assemblies.
\end{abstract}

2012 ACM Subject Classification Theory of computation; Theory of computation $\rightarrow$ Design and analysis of algorithms

Keywords and phrases optical maps, de Bruijn graph, assembly

Digital Object Identifier 10.4230/LIPIcs.WABI.2020.9

Supplementary Material Our software, RMAPPER is written in $\mathrm{C}++$ and is publicly available under GNU General Public License at https://github.com/kingufl/Rmapper.

Funding Kingshuk Mukherjee: Supported by the National Science Foundation (NSF) IIS (Grant No. 1618814).

Massimiliano Rossi: Supported by the National Science Foundation (NSF) IIS (Grant No. 1618814). Leena Salmela: Supported by Academy of Finland (grants 308030, 314170, and 323233).

Christina Boucher: Supported by the National Science Foundation (NSF) IIS (Grant No. 1618814).

(c) (i) Kingshuk Mukherjee, Massimiliano Rossi, Leena Salmela, and Christina Boucher;

under Creative Commons License CC-BY

20th International Workshop on Algorithms in Bioinformatics (WABI 2020).

Editors: Carl Kingsford and Nadia Pisanti; Article No. 9; pp. 9:1-9:16

Leibniz International Proceedings in Informatics 


\section{Introduction}

In 1993 Schwartz et al. developed optical mapping [24], a system for creating an ordered, genome wide high resolution restriction map of a given organism's genome. Since this initial development, genome wide optical maps have found numerous applications including discovering structural variations $[12,8]$, scaffolding and validating contigs for several large sequencing projects $[9,4]$, and detecting misassembled regions in draft genomes $[27,16,20]$. Thus, optical mapping has assisted in the assembly of a variety of species - including various prokaryote species $[23,31,32]$, rice [33], maize [34], mouse [6], goat [7], parrot [9], and amborella trichopoda [4]. Bionano Genomics has enabled the automated generation of the data, enabling the data to become more wide-spread. For example, Bionano data was generated for 133 species sequenced for the Vertebrate Genomes Project.

Similar to sequencing, the protocol for producing optical mapping data, begins with many fragmented copies of the genome of interest. This redundancy allows overlap between the raw data and assembly into longer contiguous regions corresponding to the genome. With a selected enzyme, the fragments are nicked at each restriction site recognized by the enzyme. These nicked fragments are then photographed and analyzed in order to determine the length (in $\mathrm{kbp}$ ) of the regions between nick sites. The result of this process are optical maps for all the fragments, which are referred to as Rmaps. For example, given a genome fragment TTTTAACTGGGGGGGAACTTTTTTTTAACTTTTT and an enzyme that recognizes the site AACT and cleaves in the middle, the resulting Rmap would be $[6,11,11,6]$. Rmaps by themselves are not traditionally used for analysis - although, they can be [17, 8, 12] - and instead have to be assembled into longer contiguous optical maps corresponding to the genome. Hence, assembly of Rmaps refers to the problem of generating a consensus genome wide optical map from overlapping Rmaps.

Although optical mapping has been around for decades now, the problem of efficiently assembling the data largely remains open as there has been little work in this area - which is largely due to the challenges posed by the data itself. Rmap data has a number of errors that make it difficult to assemble - namely, there exists added and deleted cut sites and sizing error, resulting in extra fragments, merges in neighboring fragments and under or over-estimates of the length of a fragment. In the running example, the error free Rmap of $[6,11,11,6]$ could occur as $[6,22,6]$ with error. Nonetheless, there exists two Rmap assembly methods: Gentig by Anantharaman et al. [1] and the assembler of Valouev et al. [29]. Developed in 1998, Gentig is the first Rmap assembly algorithm. It is based on a Bayesian model that seeks to maximize the a posteriori estimate of the consensus optical map produced by the assembly of Rmaps. It first computes the overlap between all pairs of Rmaps using dynamic programming, and then builds contigs by greedily merging the Rmaps based on alignment score. This process of merging contigs continues until all alignments above a certain score are merged. Valouev et al. [29] implemented an overlap-layout-consensus (OLC) assembly algorithm using their alignment algorithm [28], which also starts by calculating alignment between all pairs of Rmaps, and identifying all alignments that have score above a specified threshold. A graph is built, where Rmaps are represented as nodes, and the non-filtered alignments are represented as edges. The graph is refined by eliminating paths in the graph that are weakly supported. In other words, if two connected regions in the graph are joined by only a single path - or with multiple paths, but having one or more common intermediate nodes - then the graph is disconnected at these nodes. Further, an edge is removed if it is inconsistent with a higher scoring edge. Contigs are then generated by traversing this graph in a depth first manner. Bionano Genomics Inc. provides a proprietary assembly method, called Bionano Solve, however the source code is not publicly available and the algorithmic details are unknown due to the proprietary nature of the software. 
The alternative to an OLC approach for assembly is Eulerian assembly that relies on building and traversing the de Bruijn graph. For simplicity, we give a constructive definition of the de Bruijn graph in the context of genome assembly. Given a set of sequences $R=\left\{r_{1}, \ldots, r_{n}\right\}$ and an integer $k$, the de Bruijn graph is constructed by creating a directed edge for each unique $k$ length substring ( $k$-mer) with the nodes labeled as the $k-1$ length prefix and $k-1$ length suffix of the $k$-mer, and then all nodes that have the same label are merged. The important aspect of Eulerian assembly is that it avoids having to find alignments between any pair of sequences, leading to an $\mathcal{O}(n)$ run-time. Since its introduction by Idury et al. [11] and Pevzner et al. [22], Eulerian assembly has become the most common paradigm for assembling short read sequencing data because it led to huge gains in performance over OLC approaches. Hence, applying a Eulerian approach to Rmap assembly would likely lead to similar improvements by removing the burden of finding all pairwise alignments between Rmaps. The challenge we face is constructing a de Bruijn graph with added and deleted cut-sites and sizing error. Eulerian assembly works on the premise that a $k$-mer will occur exactly without error frequently in the data. Even without the occurrence of added and deleted cut-sites, $k$-mers created from Rmap data are unlikely to be exact replicas due to sizing error. For example, $[6,11,11,6]$ and $[5,10,11,7]$ should likely be recognized as instances of the same $k$-mers in Rmap data. Thus, to overcome this challenge the de Bruijn graph has to be redefined to account for the inexactness of the data.

In this paper, we formulate and describe an Eulerian approach for de novo Rmap assembly, which heavily relies on redefining the de Bruijn graph to make it suitable for Rmap data. We accomplish this by extending the definition of a bi-label in the context of the paired de Bruijn graph that was introduced by Medvedev et al. [14]. We refer to our modified de Bruijn graph as bi-labelled de Bruijn graph. Next, we demonstrate how to efficiently build and store the de Bruijn graph using a two tier orthogonal-range search data structure. We implement this approach, leading to a novel Rmap assembler that we call RMAPPER. We compare the performance of our method with the assembler of Valouev et al., and Bionano Solve on three genomes of varying size: E. coli, human, climbing perch (a fish species from the Vertebrate Genomes Project). Our comparison demonstrates that RMAPPER was more than 130 times faster and used less than five times less memory than Solve, and was more than 2,000 times faster than Valouev et al. Consequently, RMAPPER was the only method able to scale to the largest Rmap dataset: climbing perch. It successfully assembled the 3.1 million Rmaps of the climbing perch genome into contigs that covered over $87 \%$ of the draft genome with zero mis-assemblies.

\section{Background and definitions}

\subsection{Rmap Data and Genome Wide Optical Maps}

From a computer science perspective, we can view an $\operatorname{Rmap} R=\left[r_{1}, r_{2}, \ldots, r_{|R|}\right]$ as an ordered list of integers. Each number represents the length of the respective fragment. The size of an Rmap $R$ denotes the number of fragments in $R$, which we denote as $|R|$. For example, say we have an enzyme that cleaves the DNA at the middle position of AACT and a genomic sequence TTTTAACTGGGGGGGAACTTTTTTTTAACTTTTT, then the Rmap will be $R=[6,11,11,6]$ corresponding to the cleaved sequences [TTTTAA, CTGGGGGGGAA, СTTTTTTTTAA, СТTTTT]. 


\subsection{Error Profile of Rmap Data}

There are three types of errors that can occur in optical mapping: (1) missing cut sites which are caused by an enzyme not cleaving at a specific site, (2) additional cut sites which can occur due to random DNA breakage and (3) inaccuracy in the fragment size due to the inability of the system to accurately estimate the fragment size. Continuing again with the example above, an example of an additional cut site would be when the second fragment of $R$ is split into two, e.g., $R^{\prime}=[6,5,6,11,6]$, and an example of a missing cut site would be when the last two fragments of $R$ are joined into a single fragment, e.g., $R^{\prime}=[6,11,17]$. Lastly, an example of a sizing error would be if the size of the first fragment is estimated to be 7 rather than 6 .

Several different probabilistic models have been proposed for describing the sizing error, and the frequency of added and missed cut-sites, including the models of Valouev et al. [28], Li et al. [13], and Chen et al. [5]. We briefly describe these models here but refer to the original papers for a full description. Both Valouev et al. and Chen et al. describe the observed fragment lengths as normal distribution with the mean being equal to the true length of the fragment and the standard deviation being a function of the true length, i.e. longer fragments exhibit larger standard deviation. In the model by Li et al. the sizing error uses a Laplace distribution as follows: if the observed and actual size of a fragment are $o_{i}$ and $r_{i}$, respectively, then the sizing error, $o_{i} \sim r_{i} \times \operatorname{Laplace}(\mu, \beta)$ where $\mu$ and $\beta$ are parameters of the Laplace distribution and are functions of $r_{i}$. All studies model the probability of having a missed cut-site as a Bernoulli trial. Valouev et al. and Chen et al. predict a fixed probability for digestion of a cut-site while Li et al. model the probability of digestion as a function of lengths of the fragments flanking the cut-site. The likelihood of a missed cut-site decreases with the length of the fragment. All three models postulate additional or false cut-sites result from random breaks of the DNA molecule and hence model the number of false cuts per unit length of DNA as a Poisson distribution. Li et al. observed that false cuts occurred less frequently at the two ends of an Rmap.

\subsection{Rmap Segments and k-mers}

We define a segment $s_{p, q}$ of an Rmap starting at position $p$ and ending at position $q$, as the $q-p+1$ consecutive fragments starting from $r_{p}$, i.e., $\left[r_{p}, r_{p+1}, . ., r_{q}\right]$. We define the length of a segment as the summation of all of its constituent fragments, i.e., $r_{p}+\cdots+r_{q}$. We denote the length of a segment $s_{p, q}$ as $\ell\left(s_{p, q}\right)$. We note that the length of the Rmap $R$ should not be confused with the number of fragments, which we denote as its size $|R|$.

In this paper, we extend the definition of a $k$-mer to the context of Rmap data as follows. Given an integer $k$, we define a $k$-mer as a segment of exactly $k$ fragments, i.e., a sequence of $k$ successive fragments of an Rmap. Following the example from above, the following two 3 -mers exist in $R=[6,11,11,6]:[6,11,11]$ and $[11,11,6]$.

\subsection{Prefixes and Suffixes of Rmaps}

Given an Rmap $R=\left[r_{1}, r_{2}, \ldots, r_{|R|}\right]$, we define the $x$-size prefix of $R$ as $R=\left[r_{1}, r_{2}, \ldots, r_{x}\right]$, where $x$ is at most $|R|-1$. Conversely, we define the $x$-size suffix of $R$ as $R=\left[r_{|R|-x+1}, \ldots, r_{|R|}\right]$, where $x$ is at most $|R|-1$. 


\section{The Bi-labelled de Bruijn Graph}

In this section, we modify the traditional definition of the de Bruijn graph for Rmap data by first redefining the concept of a bi-label for Rmap data. The term bi-label was first introduced by Medvedev et al. [14] in the context of short read assembly to incorporate mate-pair data into assembly of paired-end reads. There the term bi-label refers to two $k$-mers separated by a specified genomic distance. The redefinition of the de Bruijn graph with this extra information was shown to de-tangle the resulting graph, making traversal more efficient and accurate. Here, we demonstrate that an equivalent paradigm can be effective for Rmap assembly.

\subsection{Bi-labels}

Given integers $k$ and $D$, and Rmap $R$, we define a bi-label from an Rmap $R$, as a segment of $R$ containing a pair of $k$-mers separated by the shortest segment that has a length of at least $D$. The following is a formal definition.

Definition 1. Given an Rmap $R=\left[r_{1}, r_{2}, \ldots, r_{i}, r_{i+1}, \ldots, r_{|R|}\right]$, integers $k$ and $D$, and a position $i$, we define the bi-label at position $i$ to be $\left[s_{k}^{1}, r_{p}, \ldots, r_{q}, s_{k}^{2}\right]$, where $p=i+k$ and $q$ is an index such that $\ell\left(s_{p, q-1}\right)<D \leq \ell\left(s_{p, q}\right)$ and $s_{k}^{1}$ and $s_{k}^{2}$ are the $k$-mers starting at positions $i$ and $q+1$, respectively.

Next, we refer to segment $s_{p, q}$ between $s_{k}^{1}$ and $s_{k}^{2}$ as the skip segment, and note that, unlike $s_{k}^{1}$ and $s_{k}^{2}$ which both have $k$ fragments, this segment is only bounded by its length and can have any number of fragments. Thus, this accounts for added and deleted cut-sites since these errors do not impact the length of a segment. Figure 1 demonstrates how the skip-segment tolerates a deleted cut-site.

For example, given $k=3, D=25$, and $R=[7,18,13,3,15,12,4,3,6,5,13,2]$, the bi-labels of $R$ are $([7,18,13]|[3,15,12]|[4,3,6]),([18,13,3]|[15,12]|[4,3,6])$ and $([13,3,15] \mid[12,4,3,6]$ $[5,13,2])$.

We are now going to define the prefix and suffix bi-labels.

- Definition 2. Given integers $D$ and $k$ and bi-label $b$ with $k$-mers $b^{1}=\left[b_{1}^{1}, . . b_{k}^{1}\right]$ and $b^{2}=\left[b_{1}^{2}, . ., b_{k}^{2}\right]$ and skip segment $b^{s}$, we define the prefix bi-label of $b$ as the bi-label with $(k-1)$-mers and skip-segment length at least $D$, where the first $(k-1)$-mer is the $(k-1)$-size prefix of $b^{1}$ i.e. $\left[b_{1}^{1}, . . b_{k-1}^{1}\right]$.

Note that the second $(k-1)$-mer of the prefix bi-label is not necessarily the $(k-1)$-size prefix of $b^{2}$. We also require an equivalent definition for the suffix of a bi-label.

- Definition 3. Given integers $D$ and $k$ and bi-label $b$ with $k$-mers $b^{1}=\left[b_{1}^{1}, . . b_{k}^{1}\right]$ and $b^{2}=\left[b_{1}^{2}, . ., b_{k}^{2}\right]$ and skip segment $b^{s}$, we define the suffix bi-label of $b$ as the bi-label with $(k-1)$-mers and skip-segment length at least $D$, where the first $(k-1)$-mer is the $(k-1)$-size suffix of $b^{1}$ i.e. $\left[b_{2}^{1}, . . b_{k}^{1}\right]$.

Figure 2 illustrate this concept of prefix and suffix bi-labels. Note that for two successive bi-labels from an Rmap, the prefix bi-label of the latter is the same as the suffix bi-label of the former as shown in Figure 2. This is a vital property that allows the de Bruijn graph constructed over bi-labels to be connected. 


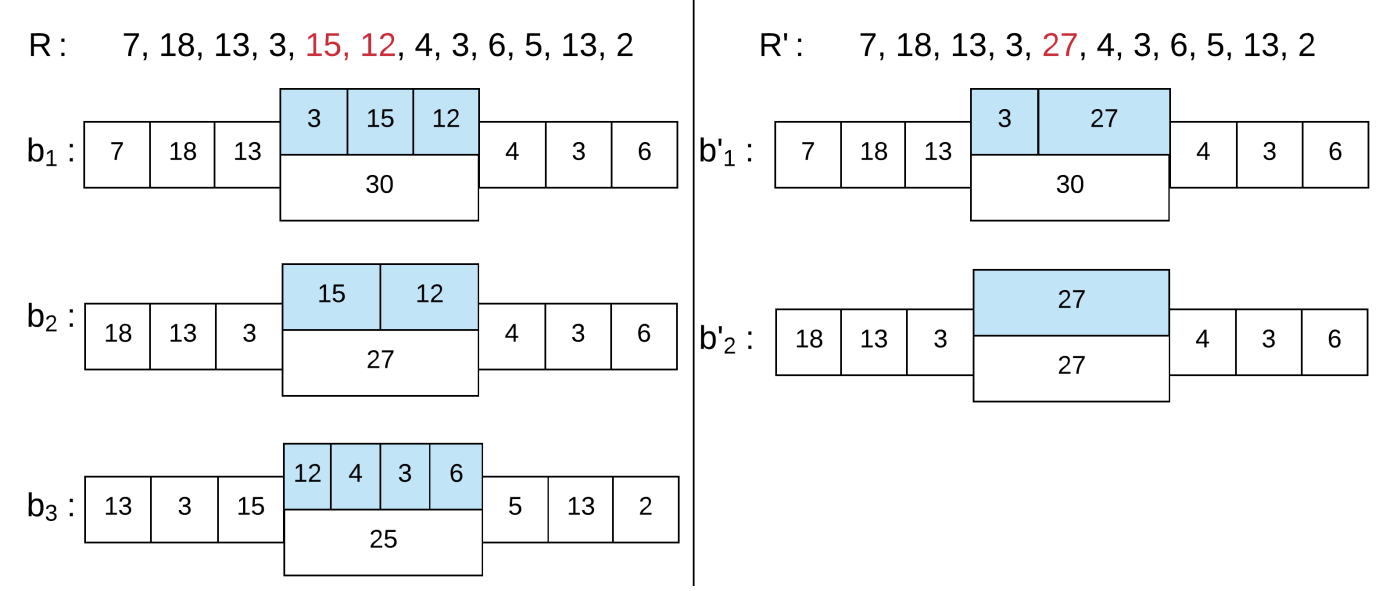

Figure 1 All bi-labels for $k=3$ and $D=25$ of two Rmaps $R$ and $R^{\prime},\left\{b_{1}, b_{2}, b_{3}\right\}$ and $\left\{b_{1}^{\prime}, b_{2}^{\prime}\right\}$ respectively. Both Rmaps cover the same genomic location but $R^{\prime}$ has a missed cut-site in position 5 (shown in red). On each bi-label the fragments from the $k$-mers and the length of the skip segment are shown in white while the fragments of the skip segment are shown in blue. Despite the missed cut-site on $R^{\prime}$ bi-labels $b_{1}$ and $b_{2}$ are merged to $b_{1}^{\prime}$ and $b_{2}^{\prime}$ respectively according to our merge function.

\subsection{Bi-label Proximity}

One of the challenges with Rmap data is the fact that the fragments correspond to genomic distances and due to experimental error, the measured estimates for the same genomic fragment are different across different Rmaps representing the same genomic location. For example, $R=[5,6,7,11,5]$ and $R^{\prime}=[6,5,6,11,6]$ likely correspond to the same $k$-mer but the numerical nature makes it such that they are not exactly equal. Thus, we need to define a criteria such that two bi-labels drawn from different Rmaps but corresponding to the same genomic locations can be identified and merged for the construction of the de Bruijn graph. Thus, to make the definition of a bi-label robust to sizing errors, we define conditions on both the difference of the individuals fragments of two bi-labels and the difference in the total lengths. Hence, we have the following definitions.

- Definition 4. Given integers $t_{f}, k$ and $D$, and two bi-labels $a$ and $b$, we let the $k$-mers of $a$ and $b$ be $a^{1}=\left[a_{1}^{1}, . ., a_{k}^{1}\right]$ and $a^{2}=\left[a_{1}^{2}, . ., a_{k}^{2}\right]$ and $b^{1}=\left[b_{1}^{1}, . ., b_{k}^{1}\right]$ and $b^{2}=\left[b_{1}^{2}, . ., b_{k}^{2}\right]$, respectively. We define $a$ and $b$ to be fragment proximal if and only if $\left|a_{i}^{1}-b_{i}^{1}\right| \leq t_{f}$ and $\left|a_{i}^{2}-b_{i}^{2}\right| \leq t_{f}$ for all $i=1, . ., k$.

Here $t_{f}$ is an error-tolerance parameter that handles sizing errors on the fragments of the bi-label.

- Definition 5. Given integers $t_{\ell}, k$ and $D$, and two bi-labels $a$ and $b$, we let the $k$-mers of $a$ and $b$ be $a^{1}$ and $a^{2}$ and $b^{1}$ and $b^{2}$, respectively, and the skip segment of $a$ and $b$ be $a^{s}$ and $b^{s}$, respectively. We define $a$ and $b$ to be length proximal if and only if $\left|\ell\left(a^{1}\right)-\ell\left(b^{1}\right)\right| \leq t_{\ell}$, $\left|\ell\left(a^{2}\right)-\ell\left(b^{2}\right)\right| \leq t_{\ell}$ and $\left|\ell\left(a^{s}\right)-\ell\left(b^{s}\right)\right| \leq t_{\ell}$.

Here $t_{\ell}$ is another error-tolerance parameter that handles sizing errors on the segment lengths of the bi-label. These two definitions lead to our final definition that defines whether two bi-labels should be defined as equivalent in the de Bruijn graph. 
- Definition 6. Given integers $k$ and $D$ and two bi-labels a and $b$, we define them to be proximal if and only if they are fragment proximal and length proximal.

This leads to our final definition, which is the set of bi-labels in which the bi-labelled de Bruijn graph is defined on.

- Definition 7. Given a set of Rmaps $\left\{R_{1}, . ., R_{n}\right\}$ and integers $k$ and $D$, let $B$ be the set of bi-labels from $R$. We define the proximal reduced set of bi-labels as the set $B^{\prime}$, where for each $b$ in $B$ there is a bi-label in $B^{\prime}$ that it is proximal to.

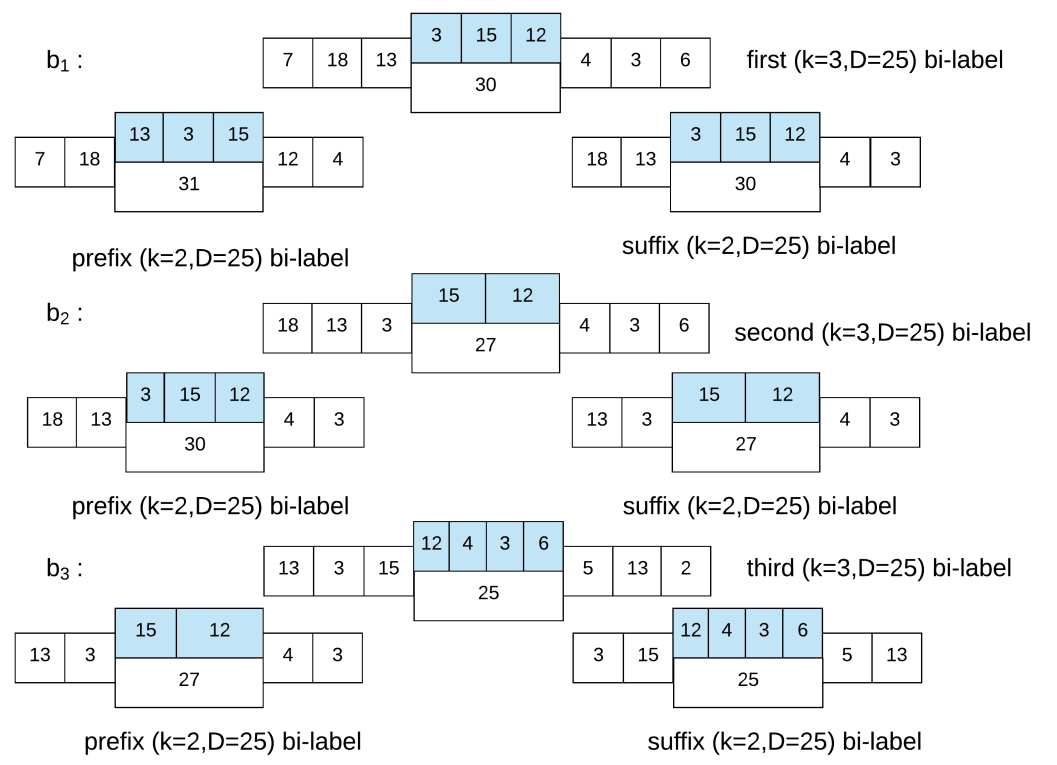

Figure 2 All bi-labels for $k=3$ and $D=25$ of an Rmap $R$. On each bi-label the fragments from the $k$-mers and the length of the skip segment are shown in white while the fragments of the skip segment are shown in blue. For each bi-label we show the prefix and suffix bi-labels built with $k=2$ and $D=25$.

\subsection{Definition of the Bi-labelled de Bruijn Graph}

Given the above definitions, we are now ready to define the bi-labelled de Bruijn graph built on a set of proximal bi-labels extracted from Rmaps.

- Definition 8. Given integers $k$ and $D$ and set of Rmaps $\left\{R_{1}, . ., R_{n}\right\}$, let $B$ be the set of proximal bi-labels extracted from $R$. We create a directed edge e for each bi-label $b$ in $B$ and label the incoming and outgoing nodes of $e$ as the prefix bi-label of $b$ and suffix bi-label of $b$, respectively. After all edges are formed, the graph undergoes a gluing operation. A pair of node bi-labels are glued into a single node if and only if they are proximal. We define the final graph obtained after gluing of nodes as the bi-labelled de Bruijn graph. 


\section{$4 \quad$ Methods}

In this section, we describe our method for building and traversing the bi-labelled de Bruijn graph from an Rmap dataset. Our method, which we refer to as RMAPPER, can be summarized into the following steps: extract and store bi-labels, find proximal bi-labels, build the bi-labelled de Bruijn graph, resolve tips and bubbles, and traverse the graph to build the contigs. We now describe each of these steps in detail.

\subsection{Extract and Store all Bi-lablels}

We first error correct the Rmap data using COMET [18] and then extract and store all bi-labels from the error corrected Rmaps. We recall from Definition 6 that two bi-labels are proximal if they are both fragment proximal as well as length proximal for error-tolerance parameters $t_{f}$ and $t_{\ell}$. Therefore, we must store all the bi-labels in a manner that allows finding all proximal bi-lablels of a given bi-label efficiently. To accomplish this, we store all the bi-labels in a disjoint set of k-d trees [3] such that each pair of bi-labels in the same k-d tree are length proximal. For each bi-label, the $2 k$ fragments of the $k$-mers of it are stored in the corresponding $\mathrm{k}$ - $\mathrm{d}$ tree, which will allow for efficiently finding all fragment proximal bi-labels of a given bi-label. Hence, the dimension of each $\mathrm{k}-\mathrm{d}$ tree is $2 k$.

More formally, we identify each k-d tree $\mathcal{K}_{a_{1}, a_{2}, a_{3}}$ by three positive integers $a_{1}, a_{2}$, and $a_{3}$, and insert a given bi-label $b$ into $\mathcal{K}_{a_{1}, a_{2}, a_{3}}$ if the length of its two $k$-mers $\ell\left(b^{1}\right)$ and $\ell\left(b^{2}\right)$ are within the range $\left[a_{1} \times t_{\ell}, \ldots,\left(a_{1}+1\right) \times t_{\ell}-1\right]$ and $\left[a_{2} \times t_{\ell}, \ldots,\left(a_{2}+1\right) \times t_{\ell}-1\right]$ respectively and the length of the skip segment $\ell\left(b^{s}\right)$ is also within the range $\left[a_{3} \times t_{\ell}, \ldots,\left(a_{3}+1\right) \times t_{\ell}-1\right]$. If such a tree does not exist then we create a new one with $\mathcal{K}_{a_{1}, a_{2}, a_{3}}$, where $a_{1}=\left\lfloor\ell\left(b^{1}\right) / t_{\ell}\right\rfloor$, $a_{2}=\left\lfloor\ell\left(b^{2}\right) / t_{\ell}\right\rfloor$ and $a_{3}=\left\lfloor\ell\left(b^{s}\right) / t_{\ell}\right\rfloor$.

Next, for each bi-label in our set of $\mathrm{k}$ - $\mathrm{d}$ trees, we find and store pointers to all proximal bi-labels by performing an orthogonal range query. Given a bi-label $b$ in $\mathcal{K}_{a_{1}, a_{2}, a_{3}}$, we let the $k$-mers of the bi-label $b$ be $b^{1}=\left[b_{1}^{1}, . ., b_{k}^{1}\right]$ and $b^{2}=\left[b_{1}^{2}, . ., b_{k}^{2}\right]$. We perform a range query with $\left(\left[b_{1}^{1} \pm t_{f}\right], \ldots,\left[b_{k}^{1} \pm t_{f}\right],\left[b_{1}^{2} \pm t_{f}\right], \ldots,\left[b_{k}^{2} \pm t_{f}\right]\right)$ in the disjoint set of $\mathrm{k}$-d trees to find all bi-labels whose first $k$-mer is equal to $\left[b_{1}^{1} \pm t_{f}\right], \ldots,\left[b_{k}^{1} \pm t_{f}\right]$ and whose second $k$-mers is equal to $\left[b_{1}^{2} \pm t_{f}\right], \ldots,\left[b_{k}^{2} \pm t_{f}\right]$. We add a pointer from $b$ to each of these bi-labels. We repeat this for each bi-label. In particular, we perform the range query in all k-d trees where the proximal bi-labels can be found, i.e., all k-d trees $\mathcal{K}_{a_{1}^{\prime}, a_{2}^{\prime}, a_{3}}$ where for $m=\min \left(k t_{f}, t_{\ell}\right)$ we have, $\left\lfloor\left(\ell\left(b^{1}\right)-m\right) / t_{\ell}\right\rfloor \leq a_{1}^{\prime} \leq\left\lfloor\left(\ell\left(b^{1}\right)+m\right) / t_{\ell}\right\rfloor$ and $\left\lfloor\left(\ell\left(b^{2}\right)-m\right) / t_{\ell}\right\rfloor \leq a_{2}^{\prime} \leq\left\lfloor\left(\ell\left(b^{2}\right)+m\right) / t_{\ell}\right\rfloor$.

We note that $\mathrm{k}-\mathrm{d}$ trees support multi-dimensional orthogonal range-search queries in $\mathcal{O}\left(n^{(2 k-1) / 2 k}+o c c\right)$ time and $\mathcal{O}(n)$ space where $n$ is the number of bi-labels in the tree, $k$ is the $k$-mer value, and occ is the number of bi-labels that satisfy the constrains of the range-search query.

\subsection{Graph Construction}

We first filter all low frequency bi-labels, i.e., bi-labels that have a low number of proximal bi-labels. As illustrated in Figure 3, bi-labels that have low frequency typically arise from Rmap data that is highly erroneous. After filtering low frequency bi-labels, we build the bi-labelled de Bruijn graph by first building a proximal reduced set from the unfiltered bi-labels, then building all directed edges with labelled nodes from the reduced set, and finally merging nodes that have the same label. Using an efficient heuristic, we first greedily find the proximal reduced set of bi-labels by sorting the unfiltered bi-labels in descending order based on the number of proximal bi-labels found for them. From this sorted list of bi-labels $B$, we iteratively insert bi-labels into the reduced set $B^{\prime}$ unless the bi-label is proximal to a bi-label already in $B^{\prime}$. 


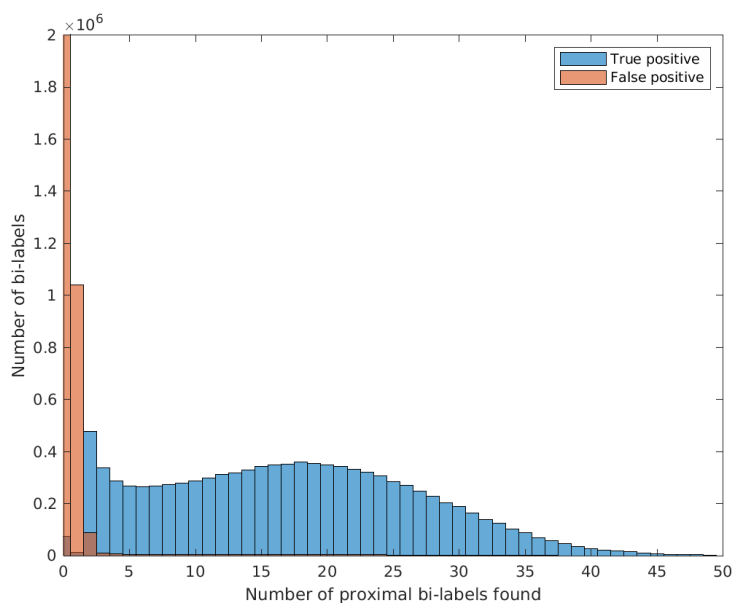

Figure 3 Histogram showing the precision of finding proximal bi-labels. For simulated human Rmap data, we found proximal bi-labels for all extracted bi-labels. We designate a proximal bi-label found to be a true positive if its true location in the genome is the same as the location of the bi-label to which it is proximal - and false positive otherwise. Next, we plotted a histogram showing the distribution of true positives and false positive proximal bi-labels for each bi-label. We show that high frequency bi-labels i.e. bi-labels for which we find more proximal bi-labels produce more precise proximal bi-labels. This justifies filtering low frequency bi-labels.

Next, we build a bi-labelled de Bruijn graph by creating a directed edge for each bi-label $b^{\prime}$ in $B^{\prime}$ and labeling the incoming and outgoing nodes as the prefix bi-label and suffix bi-label of $b^{\prime}$. We store all the nodes and edges in a modified adjacency list format that contains three arrays: one array stores all node bi-labels, one array containing a list of pointers of the incoming nodes for each node, and lastly, one array containing a list of pointers of the outgoing nodes for each node. Thus, to insert $b^{\prime}$ into the graph, we first determine if the prefix and suffix bi-labels are contained in the node array and insert them if they are not contained in the list, and then insert an entry into the incoming and outgoing arrays with lists containing pointers to the prefix and suffix bi-labels. This graph representation will allow for the adjacency lists of two nodes to be efficiently merged if the bi-labels they represent are found to be proximal.

Lastly, we merge all nodes in the graph whose bi-labels are proximal to obtain the final bi-labelled de Bruijn graph. For merging the nodes, we again use a set of disjoint k-d trees as we did before for finding proximal bi-labels for the edge bi-labels. Hence, we extract all the node bi-labels and construct a set of $\mathrm{k}-\mathrm{d}$ trees as before. Then for each node $v$ in the node array, we query the corresponding $\mathrm{k}-\mathrm{d}$ trees to find all nodes that are proximal to it using the same error tolerance parameters $t_{f}$ and $t_{\ell}$. Any node $u$ that is found to be proximal to $v$ is merged to $v$ by removing $u$ from the graph by updating the two adjacency lists such that the incoming and outgoing array entries storing pointers to $u$ are updated to store pointers to $v$. This can be achieved in linear time. We repeat this until all proximal nodes have been merged. Figure 4 illustrates the construction of the bi-labelled de Bruijn graph for a pair of Rmaps.

\subsection{Graph Cleaning and Traversal}

Before traversing the graph, we first pre-process the bi-labelled de Bruijn graph to remove tips and bubbles, which are common in de Bruijn graphs. Since they limit the size of unary paths (i.e. paths in the graph that contain nodes with only a single outgoing edge) and 
$\mathrm{R}_{1}: 7,18,13,3,15,12,4,3,6,5,13,2$

$R_{2}: 7,18,13,3,27,4,3,6,5,13,2$

(a)

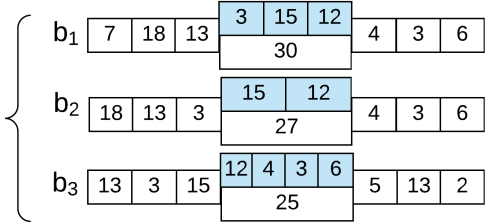

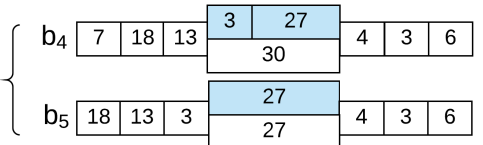

(d)

Figure 4 The construction of the bi-labelled de Bruijn Graph. (a) Two Rmaps $R_{1}$ and $R_{2}$ and the bi-labels extracted from them $-\left\{b_{1}, b_{2}, b_{3}\right\}$ from $R_{1}$ and $\left\{b_{3}, b_{4}\right\}$ from $R_{2}$ for $k=3$ and $D=25$. (b) Edges $\left\{e_{1}, e_{2}, e_{3}\right\}$ depict the proximal reduced set of bi-labels. Bi-labels $\left\{b_{1}, b_{4}\right\}$ are represented by $e_{1}$, bi-labels $\left\{b_{2}, b_{5}\right\}$ are represented by $e_{2}$ and bi-label $\left\{b_{3}\right\}$ forms $e_{3}$. We note that in this example no bi-labels are filtered for finding the proximal reduced set. (c) Nodes introduced into the graph. Each edge breaks into two nodes - one denoted by the prefix bi-label and the other by suffix bi-label of the edge. A directed edge is drawn from the former to the latter. (d) The final graph is formed by merging nodes $v_{12}$ with $v_{21}$ and merging $v_{22}$ with $v_{32}$.

do not affect the accuracy of the assembly, it is common practice in short read assembly to resolve or remove these structures $[2,30,26,21]$. Tips are produced when errors cause an otherwise unary path to branch at a node and create a short unary path that ends in a terminal node. Bubbles are created when bi-labels from the same genomic location are not merged and included in the graph as separate edges. This generates short unary paths that have the same starting node and the same ending node and are close in length.

Similar to existing short read assemblers, we identify all tips and bubbles that have length of at most a specified threshold by performing depth first search starting at each node with out-degree greater than one. Hence, if there exists a tip starting at a given node as well as a path of length longer than the specified threshold, then the tip is removed by deleting all of its edges starting at the branching node. Furthermore, if there exists a bubble starting at a given node, we remove one of the edges adjacent to the branching node. We note we do not remove an entire path from the graph to resolve a bubble - rather, we only disconnect them at the branching node. Following the work of Simpson et al. [26], we fix the maximum length of the paths in a bubble to twice the size of the bi-label.

After cleaning, our traversal algorithm extracts unitigs (i.e. contigs corresponding to unary paths) from the graph by performing a simple depth first traversal starting from each node with zero incoming edges. We terminate the traversal of a given path if a cycle is reached or a node with out-degree greater than one is reached. 


\section{Experiments}

In this section, we compare the performance of RMAPPER, the assembler of Valouev et al. and Bionano Solve. We used the most recent version of Bionano Solve that is publicly available (version 3.5.1.). We performed all experiments on Intel E5-2698v3 processors with 192 GB of RAM running 64-bit Linux. Valouev and RMAPPER were ran on error corrected data. Bionano Solve was not because the input is required to be specified in their proprietary format. In addition, for larger genomes, we also ran RMAPPER by extracting bi-labels from both directions in an Rmap. We refer to this as RMAPPER2.0.

For all experiments we report the run time (CPU time), peak memory, maximum and mean contig size, genome fraction and number of mis-assembled contigs. We note that genome assembly evaluation tools such as QUAST [10] cannot be used on optical maps - hence, we design our own evaluation setup. To compute the genome fraction, we align all assembled contigs to the optical map reference genome using the alignment method of Valouev et al. [28]. The optical map reference genome is produced by in silico digesting the reference genome using the same restriction enzyme as used for producing the Rmaps. For all contigs that were successfully aligned, we designate their alignment locations on the reference genome as covered and report the percentage of the genome covered by at least one contig as the genome fraction. Any contig which is unable to be aligned by Valouev et al. is verified to be mis-assembled by aligning it to the reference genome using a second alignment software - Bionano's RefAligner. The Valouev method aligns an assembled contig to a contiguous stretch of the reference optical map that optimizes its alignment score and does not tolerate mis-assembled regions. Whereas, RefAligner allows split alignments. Hence, if the alignment outputted from RefAligner is uncontiguous then it is counted as a mis-assembly.

RMAPPER takes as input four parameters, namely the size $k$ of the $k$-mers, the minimum distance $D$ between the two $k$-mers in the bi-label, and the error tolerance parameter setting $t_{f}$ and $t_{\ell}$. The $k$-mer size depends on the error-rate of the Rmap data. When the frequency of added and missed cut-sites is high, the $k$-mer size needs to be set low so that a good percentage of $k$-mers are error-free. We node that the average error-rate of optical-map data typically lies between $14 \%$ to $16 \%$. Considering that error-correcting the Rmaps brings the average error-rate below $10 \%$, the $k$-mer size of 6 is the largest value such that the probability that an extracted $k$-mer will be error-free is at least $50 \%$. Hence we use 6 as the default $k$-mer size in our experiments. The best combination of coverage, average length of contigs and run-time is achieved by fixing $t_{\ell}=2000$. We experimented with the following values of $D=\{15000,20000,25000,30000\}$ and the following values of $t_{f}=\{500,1000,1500\}$ and for each experiment, we choose the parameter setting that gives the best performance. A higher value of $t_{f}$ is needed when the Rmap data still has significant sizing errors after error correction. A lower value of $D$ is needed when the average Rmap size is small so that we can extract an adequate number of bi-labels from each Rmap.

\subsection{Datasets}

We performed experiments on both simulated and real Bionano datasets. We simulated data from both $E$. coli K-12 substr. MG1655 genome and the human reference genome GRCh38 (NCBI accession number GCF_000001405.26) with OMSim [15]. We used enzyme BspQI - a standard, commonly used restriction enzyme for optical mapping - and used the default error rate of OMSim, which is a $15 \%$ rate of deleted cut sites, and 1 added cut site per $100 \mathrm{kbp}$. The resulting E. coli dataset contains 23450 Rmaps with a mean of 42 fragments per Rmap. The Human dataset contains 377894 Rmaps with a mean of 61 fragments per Rmap. 
Lastly, we performed experiments using the Rmap dataset of the climbing perch (Anabas testudineus) genome generated for the Vertebrate Genomes Project, which consists of 3121480 Rmaps with mean of 28 fragments. A draft assembly of the genome is provided from the same source which was used to obtain the reference genome optical map.

\subsection{Performance on E. coli}

The results on E. coli Rmap dataset is summarized in Table 1. For this experiment we extracted bi-labels with $k=6$ and $D=15000$ and used error tolerance parameter setting $t_{f}=500$ and $t_{\ell}=2000$. RMAPPER took 342 seconds and peak memory of $274 \mathrm{Mb}$ to assemble the data. The assembler produced two unitigs that are 529 and 522 fragments in length, which covered the reference from start to finish.

Table 1 Assembly results for E. coli Rmap data simulated by OMSim using enzyme BspQI. The dataset has 23,450 Rmaps of mean size of 42 fragments and coverage of 900x. The peak memory is given in gigabytes (GB). The run time is reported in second $(\mathrm{s})$ minutes $(\mathrm{m})$, hours $(\mathrm{h})$ and days (d). RMAPPER was run with $k=6, D=15000$ and error tolerance parameter setting $t_{f}=500$ and $t_{\ell}=2000$. The contig with maximum length (Max) is reported in the number of fragments and the total genomic length in mega base pairs (Mbp). Similarly, the mean contig length (Mean) is also reported in the number of fragments and the total genomic length in mega base pairs. The genome fraction (GF) is the percentage of the genome that is covered by at least one contig. Lastly, the number of mis-assembled contigs (MA) is given.

\begin{tabular}{cccccccc} 
Assembler & Run time & $\begin{array}{c}\text { Peak } \\
\text { Memory }\end{array}$ & $\begin{array}{c}\text { No. of } \\
\text { contigs }\end{array}$ & Max & Mean & GF(\%) & MA \\
\hline Valouev & $8.5 \mathrm{~d}$ & 0.48 & 5 & $\begin{array}{c}102 \\
(1.0 \mathrm{Mbp})\end{array}$ & $\begin{array}{c}56 \\
(0.5 \mathrm{Mbp})\end{array}$ & 48 & 0 \\
\hline Solve & $48.1 \mathrm{~h}$ & 1.18 & 1 & $\begin{array}{c}631 \\
(4.9 \mathrm{Mbp})\end{array}$ & $\begin{array}{c}631 \\
(4.9 \mathrm{Mbp})\end{array}$ & 100 & 0 \\
\hline RMAPPER & $6 \mathrm{~m}$ & 0.46 & 2 & $\begin{array}{c}529 \\
(4.6 \mathrm{Mbp})\end{array}$ & $\begin{array}{c}526 \\
(4.5 \mathrm{Mbp})\end{array}$ & 100 & 0 \\
\hline
\end{tabular}

The Valouev assembler [29] took 204.8 hours to compute pairwise alignments between all pairs of Rmaps and an additional 30 minutes to assemble them into contigs. It produced 5 contigs with the longest contig of length 102 fragments (corresponding to a $1 \mathrm{Mbp}$ genomic span). We aligned the assembled contigs back to the reference and found the total genome coverage to be $48 \%$. Bionano solve produced a high quality assembly, i.e., one contig that spanned $100 \%$ of the genome. The assembly took 48.14 hours of CPU time (59.75 minutes of wall time using 60 CPUs in parallel) and peak memory of 1.18 GB. The Valouev aligner reported alignments for all contigs, hence we report zero mis-assembled contigs for all three methods.

In summary, the quality of Bionano Solve and RMAPPER were comparable, yet RMAPPER was 480 times faster (6 minutes versus 2889 minutes) and used less than $500 \mathrm{Mb}$ of memory.

\subsection{Performance on Human}

The results on the Human Rmap dataset are shown in Table 2. For this experiment we extracted bi-labels with $k=6$ and $D=25000$ and used error tolerance parameter setting $t_{f}=1500$ and $t_{\ell}=2000$. RMAPPER took 12.1 hours and peak memory of 7.9 GB to assemble the data whereas RMAPPER 2.0 took 22.2 hours and 18.8 GB of peak memory. RMAPPER produced 3134 contigs whereas RMAPPER 2.0 produced 2867 contigs. The maximum size 
unitig produced by RMAPPER and RMAPPER 2.0 was 1380 and 1752 fragments in length, respectively. Lastly,. RMAPPER achieved a net coverage of $95.8 \%$ while RMAPPER2.0 was able to cover $96.7 \%$ of the genome - both with zero mis-assembled contigs.

Table 2 Assembly results for human Rmap data simulated by OMSim using enzyme BspQI. The dataset has 377894 Rmaps of mean size of 61 fragments and coverage 80x. See Table 1 for a description of the assembly statistics and notation. As described in the text, RMAPPER 2.0 extracts bi-labels from Rmaps in both forward and reverse directions.

\begin{tabular}{cccccccc} 
Assembler & $\begin{array}{c}\text { Run } \\
\text { time }\end{array}$ & $\begin{array}{c}\text { Peak } \\
\text { Memory }\end{array}$ & $\begin{array}{c}\text { No. of } \\
\text { contigs }\end{array}$ & Max & Mean & GF(\%) & MA \\
\hline Valouev & $>360 \mathrm{~d}$ & $\mathrm{n} / \mathrm{a}$ & $\mathrm{n} / \mathrm{a}$ & $\mathrm{n} / \mathrm{a}$ & $\mathrm{n} / \mathrm{a}$ & $\mathrm{n} / \mathrm{a}$ & $\mathrm{n} / \mathrm{a}$ \\
\hline Solve & $122.4 \mathrm{~d}$ & 94.8 & 169 & $\begin{array}{c}14,133 \\
(124.6 \mathrm{Mbp})\end{array}$ & $\begin{array}{c}2,036 \\
(16.4 \mathrm{Mbp})\end{array}$ & 93.8 & 4 \\
\hline RMAPPER & $12.1 \mathrm{~h}$ & 7.9 & 3865 & $\begin{array}{c}1,380 \\
(14.4 \mathrm{Mbp})\end{array}$ & $\begin{array}{c}144 \\
(1.4 \mathrm{Mbp})\end{array}$ & 95.8 & 0 \\
\hline RMAPPER 2.0 & $22.2 \mathrm{~h}$ & 18.8 & 3524 & $\begin{array}{c}1,752 \\
(18.5 \mathrm{Mbp})\end{array}$ & $\begin{array}{c}203 \\
(2.0 \mathrm{Mbp})\end{array}$ & 96.7 & 0 \\
\hline
\end{tabular}

The Valouev assembler did not produce any output after $360 \mathrm{CPU}$ days so n/a is reported in Table 2. Bionano Solve produced comparably fewer but longer contigs to RMAPPER but had 4 mis-assembled contigs. In addition, it took approximately 2937 CPU hours (55 hours of wall time using $60 \mathrm{CPUs}$ in parallel) and peak memory of $94.8 \mathrm{~GB}$. It is also worth noting that Bionano Solve performs an elaborate scaffolding and stitching of contigs, which explains the relatively few number of contigs but higher mis-assembly rate. The scaffolding and stitching cannot be decoupled from the assembly since Bionano only distributed a single executable that runs both. The source code is not publicly available.

In summary, the Valouev assembler did not scale to the human genome, RMAPPER2.0 produced slightly longer contigs than RMAPPER, Bionano Solve produced the longest contigs but covered $93.8 \%$ of the genome and had 4 mis-assembled contigs. In addition, RMAPPER 2.0 has the highest genome fraction, which is $96.7 \%$. Lastly, RMAPPER and RMAPPER2.0 was 242 and 132 times faster than Solve, respectively, and used 5 times less memory.

\subsection{Performance on Climbing Perch}

The results on the climbing perch (Anabas Testudineus) Rmap dataset are shown in Table 3. For this experiment we extracted bi-labels with $k=6$ and $D=15000$ and used error tolerance parameter setting $t_{f}=1500$ and $t_{\ell}=2000$. RMAPPER took 7.5 hours and peak memory of 9.7 GB to assemble the data whereas RMAPPER 2.0 took 14.9 hours and 18.77 GB of peak memory. RMAPPER produced 1848 contigs whereas RMAPPER 2.0 produced 2489 contigs. The maximum size unitig produced by RMAPPER and RMAPPER 2.0 was 217 and 294 fragments in length, respectively. Lastly, RMAPPER achieved a genome fraction of $78.2 \%$, while RMAPPER 2.0 was able to cover $87.6 \%$ of the genome. Both RMAPPER and RMAPPER 2.0 produced zero mis-assemblies.

The Valouev assembler did not halt on this dataset after $360 \mathrm{CPU}$ days so we do not report any results. Solve was also unable to assemble this genome as it halted with a fatal error message after $156 \mathrm{CPU}$ days and using a peak memory of $16 \mathrm{~GB}$. In summary, RMAPPER was only method able to assemble this genome. Although RMAPPER and RMAPPER2.0 both successfully assembled the genome with zero mis-assemblies, RMAPPER 2.0 produced slightly longer contigs than RMAPPER and achieved a higher genome coverage. 
Table 3 Assembly results for the Rmap dataset of the climbing perch genome generated for the Vertebrate Genomes Project, which consists of 3121480 Rmaps with mean of 28 fragments. The restriction enzyme used in the experiment is BspQI. See Table 1 for a description of the assembly statistics and notation. As described in the text, RMAPPER2.0 extracts bi-labels from Rmaps in both forward and reverse directions.

\begin{tabular}{cccccccc} 
Assembler & $\begin{array}{c}\text { Run } \\
\text { time }\end{array}$ & $\begin{array}{c}\text { Peak } \\
\text { Memory }\end{array}$ & $\begin{array}{c}\text { No. of } \\
\text { contigs }\end{array}$ & Max & Mean & GF(\%) & MA \\
\hline RMAPPER & $7.5 \mathrm{~h}$ & 9.7 & 1848 & $\begin{array}{c}217 \\
(1.6 \mathrm{Mbp})\end{array}$ & $\begin{array}{c}52 \\
(0.4 \mathrm{Mbp})\end{array}$ & 78.2 & 0 \\
\hline RMAPPER2.0 & $14.9 \mathrm{~h}$ & 18.8 & 2489 & $\begin{array}{c}294 \\
(2.4 \mathrm{Mbp})\end{array}$ & $\begin{array}{c}65 \\
(0.6 \mathrm{Mbp})\end{array}$ & 87.6 & 0 \\
\hline
\end{tabular}

\section{Conclusion and Future Work}

Assembly of Rmap data is a fundamental problem in optical mapping that still remains in a nascent stage - as prior to this work, there was only a single other non-proprietary assembler. In this paper, we formulate and describe the first Eulerian approach for Rmap assembly by redefining the de Brujn graph to adapt it to Rmap data. We accomplish this by extending the definition of a bi-label introduced in the context of the paired-end de Bruijn graph by Medvedev et al. [14]. We refer to our modified de Bruijn graph as the bi-labelled de Bruijn graph and demonstrate how to efficiently build and store it using a two-tiered orthogonal range search data-structure.

We implement our approach and show its performance on multiple simulated and real datasets. Our experimental results show the only non-proprietary method (i.e. by Valouev et al. [29]) is unable to scale to the human genome, and that our method is at least 130 times faster than Bionano Solve and its memory usage is less than $20 \%$ of the memory usage of Bionano Solve. An important note about the comparison of the assemblers is that RMAPPER has a very simple traversal algorithm and does not use any sort of scaffolding. This is due to the fact that the main contribution of this work is formulating and solving the assembly of Rmaps. Bionano Solve has a scaffolding algorithm that cannot be decoupled from the assembly step since only an executable is available. Thus, the results really compare RMAPPER's unitigs with Solve's scaffolds, and RMAPPER are still comparable. This work does open the door for improving Rmap assembly by employing more involved graph traversal and/or adapting methods designed for scaffolding and stiching sequence contigs using optical mapping data $[19,25]$.

\section{References}

1 Thomas S. Anantharaman, Bud Mishra, and David C. Schwartz. Genomics via optical mapping iii: Contiging genomic DNA and variations (extended abstract). In $I S M B-99$, pages 18-27. AAAI Press, 1997.

2 Anton Bankevich et al. SPAdes: A New Genome Assembly Algorithm and its Applications to Single-Cell Sequencing. J. Comput. Biol., 19(5):455-477, 2012.

3 Jon Louis Bentley. Multidimensional binary search trees used for associative searching. Commun. ACM, 18(9):509-517, 1975.

4 Srikar Chamala et al. Assembly and validation of the genome of the nonmodel basal angiosperm amborella. Science, 342(6165):1516-1517, 2013.

5 Ping Chen, Xinyun Jing, Jian Ren, Han Cao, Pei Hao, and Xuan Li. Modelling BioNano optical data and simulation study of genome map assembly. Bioinformatics, 34(23):3966-3974, 2018 . 
6 Deanna M. Church et al. Lineage-specific biology revealed by a finished genome assembly of the mouse. PLoS Biol., 7(5):e1000112+, 2009.

7 Yang Dong et al. Sequencing and automated whole-genome optical mapping of the genome of a domestic goat (Capra hircus). Nat. Biotechnol., 31:135-141, 2013.

8 Xian Fan, Jie Xu, and Luay Nakhleh. Detecting large indels using optical map data. In Proc. of RECOMB-CG, pages 108-127, 2018.

9 Ganeshkumar Ganapathy et al. De novo high-coverage sequencing and annotated assemblies of the budgerigar genome. GigaScience, 3:11, 2014.

10 Alexey Gurevich, Vladislav Saveliev, Nikolay Vyahhi, and Glenn Tesler. QUAST: quality assessment tool for genome assemblies. Bioinformatics, 29(8):1072-1075, 2013.

11 Ramana M. Idury and Michael S. Waterman. A new algorithm forDNA sequence assembly. $J$. Comput. Biol, 2(2):291-306, 1995.

$12 \mathrm{Le} \mathrm{Li}$ et al. OMSV enables accurate and comprehensive identification of large structural variations from nanochannel-based single-molecule optical maps. Genome Biol., 18(1):230, 2017.

13 Menglu Li et al. Towards a more accurate error model for BioNano optical maps. In Proc of ISBRA, pages 67-79, 2016.

14 Paul Medvedev, Son Pham, Mark Chaisson, Glenn Tesler, and Pavel Pevzner. Paired de Bruijn graphs: a novel approach for incorporating mate pair information into genome assemblers. $J$. Comput. Biol, 18, 2011.

15 Giles Miclotte, Stéphane Plaisance, Stephane Rombauts, Yves Van de Peer, Pieter Audenaert, et al. OMSim: a simulator for optical map data. Bioinformatics, pages 2740-2742, 2017.

16 Martin D. Muggli, Simon J. Puglisi, Roy Ronen, and Christina Boucher. Misassembly detection using paired-end sequence reads and optical mapping data. Bioinformatics, 31(12):i80-i88, 2015.

17 Kingshuk Mukherjee, Bahar Alipanahi, Tamer Kahveci, Leena Salmela, and Christina Boucher. Aligning optical maps to de Bruijn graphs. Bioinformatics, 35(18):3250-3256, 2019.

18 Kingshuk Mukherjee, Darshan Washimkar, Martin D. Muggli, Leena Salmela, and Christina Boucher. Error correcting optical mapping data. GigaScience, 7, 2018.

19 Weihua Pan, Tao Jiang, and Stefano Lonardi. OMGS: optical map-based genome scaffolding. J. Comput. Biol, 27(4):519-533, 2020.

20 Weihua Pan and Stefano Lonardi. Accurate detection of chimeric contigs via BioNano optical maps. Bioinformatics, 35(10):1760-1762, 2018.

21 Yu Peng, Henry CM Leung, Siu-Ming Yiu, and Francis YL Chin. IDBA-UD: A de novo assembler for single-cell and metagenomic sequencing data with highly uneven depth. Bioinformatics, 28(11):1420-1428, 2012.

22 Pavel A. Pevzner, Haixu Tang, and Michael S. Waterman. An Eulerian path approach to DNA fragment assembly. Proc. Natl. Acad. Sci., 98(17):9748-9753, 2001.

23 Susan Reslewic et al. Whole-genome shotgun optical mapping of Rhodospirillum Rubrum. Appl. Environ. Microbiol., 71(9):5511-5522, 2005.

24 David C. Schwartz, Xiaojun Li, Luis I Hernandez, Satyadarshan P. Ramnarain, Edward J. Huff, and Yu-Ker Wang. Ordered restriction maps of saccharomyces cerevisiae chromosomes constructed by optical mapping. Science, 262:110-114, 1993.

25 Jennifer M. Shelton, Michelle C. Coleman, Nic Herndon, Nanyan Lu, Ernest T. Lam, Thomas Anantharaman, Palak Sheth, and Susan J. Brown. Tools and pipelines for BioNano data: molecule assembly pipeline and fasta super scaffolding tool. BMC Genomics, 16(1):734, 2015.

26 Jared T. Simpson, Kim Wong, Shaun D. Jackman, Jacqueline E. Schein, Steven J. M. Jones, and Inanç Birol. ABySS: a parallel assembler for short read sequence data. Genome Res. 19(6):1117-1123, 2009.

27 Brian Teague et al. High-resolution human genome structure by single-molecule analysis. Proc. Natl. Acad. Sci., 107(24):10848-10853, 2010.

28 Anton Valouev et al. Alignment of optical maps. J. Comp. Biol., 13(2):442-462, 2006. 
29 Anton Valouev, David C. Schwartz, Shiguo Zhou, and Michael S. Waterman. An algorithm for assembly of ordered restriction maps from single dna molecules. Proc. Natl. Acad. Sci., 103(43):15770-15775, 2006.

30 Daniel R. Zerbino and Ewan Birney. Velvet: Algorithms for de novo short read assembly using de Bruijn graphs. Genome Res., 18(5):821-829, 2008.

31 Shiguo Zhou et al. A whole-genome shotgun optical map of Yersinia pestis strain KIM. Appl. Environ. Microbiol., 68(12):6321-6331, 2002.

32 Shiguo Zhou et al. Shotgun optical mapping of the entire leishmania major Friedlin genome. Mol. Biochem. Parasitol., 138(1):97-106, 2004.

33 Shiguo Zhou et al. Validation of rice genome sequence by optical mapping. BMC Genom., $8(1): 278,2007$.

34 Shiguo Zhou et al. A Single Molecule Scaffold for the Maize Genome. PLoS Genetics, 5:e1000711, 2009. 